\title{
Higher-Order Linear Differential Systems with Truncated Coefficients
}

\author{
S.A. Abramov ${ }^{1, \star}$, M.A. Barkatou ${ }^{2}$, and E. Pflügel ${ }^{3}$ \\ ${ }^{1}$ Computing Centre of the Russian Academy of Sciences, \\ Vavilova, 40, Moscow 119333, Russia \\ sergeyabramov@mail.ru \\ 2 Institut XLIM, Département Mathématiques et Informatique, Université de \\ Limoges ; CNRS, 123, Av. A. Thomas, 87060 Limoges cedex, France \\ moulay . barkatou@unilim.fr \\ 3 Faculty of CISM, Kingston University, Penrhyn Road, Kingston upon \\ Thames, Surrey KT1 2EE, United Kingdom \\ E.Pfluegel@kingston.ac.uk
}

\begin{abstract}
We consider the following problem: given a linear differential system with formal Laurent series coefficients, we want to decide whether the system has non-zero Laurent series solutions, and find all such solutions if they exist. Let us also assume we need only a given positive integer number $l$ of initial terms of these series solutions. How many initial terms of the coefficients of the original system should we use to construct what we need?

Supposing that the series coefficients of the original systems are represented algorithmically, we show that these questions are undecidable in general. However, they are decidable in the scalar case and in the case when we know in advance that a given system has an invertible leading matrix. We use our results in order to improve some functionality of the Maple 17] package ISOLDE [1].
\end{abstract}

\section{Introduction}

Linear differential systems with variable (e.g. power series) matrix coefficients appear in many areas of mathematics. Laurent series solutions of such systems form a building block for other types of solutions, and more generally, algorithms for finding Laurent series solutions may be a part of various computer algebra algorithms (see e.g. [16]).

Let a linear differential system with formal Laurent series coefficients be given. First of all, we want to decide whether or not the given system has non-zero Laurent series solutions. Suppose that such solutions exist, and we need only a given positive integer number $l$ of initial terms of each of them. Then the following question arises: how many initial terms of the coefficients of the original system should we use to find what we need? Is it possible to compute at least

\footnotetext{
* Supported by RFBR grant 10-01-00249-a. 
an upper bound for the number of such terms? If we have such bound, then we can truncate the series involved into the original system before finding the truncated solutions. Such truncation leads to a system whose matrix coefficients are polynomials. In many cases it is much easier to work with such kind of systems than with systems with series matrix coefficients.

Let $k$ be a field of characteristic 0 . We denote by $k[[x]]$ the ring of formal power series with coefficients in $k$ and $k((x))=k[[x]]\left[x^{-1}\right]$ its quotient field. If $i \in \mathbb{Z}, u \in k((x))$ then the notation $\left[x^{i}\right] u$ is used for the coefficient of $x^{i}$ in $u$. For a nonzero element $u=\sum u_{i} x^{i}$ of $k((x))$ we denote by $\operatorname{val}_{x} u$ the $x$-adic valuation of $u$ defined by $\operatorname{val}_{x} u=\min \left\{i\right.$ such that $\left.u_{i} \neq 0\right\}$. By convention $\operatorname{val}_{x} 0=\infty$. For $M(x) \in \operatorname{Mat}_{m}(k((x)))$ we define $\operatorname{val}_{x} M(x)$ as the minimum of the valuations of the entries of $M(x)$.

We shall write $\theta$ for $x \frac{d}{d x}$ and consider differential systems of the form

$$
A_{r}(x) \theta^{r} y+A_{r-1}(x) \theta^{r-1} y+\cdots+A_{0}(x) y=0
$$

where $y=\left(y_{1}, y_{2}, \ldots, y_{m}\right)^{T}$ is a column vector of unknown functions of $x$. For the coefficient matrices

$$
A_{0}(x), A_{1}(x), \ldots, A_{r}(x)
$$

we have $A_{i}(x) \in \operatorname{Mat}_{m}(k[[x]]), A_{0}(x), A_{r}(x)$ are non-zero and $\min _{i}\left\{\operatorname{val}_{x}\left(A_{i}\right)\right\}=$ 0 .

Let a system $S$ be of the form (11) and define the l-truncation $S^{\langle l\rangle}$ which is obtained by omitting all the terms of degree larger than or equal to $l$ in the coefficients of $S$.

In this paper, we are concerned with two problems:

Problem 1 (Existence Problem). Given a system $S$ of the form (1), decide whether or not this system has a solution in $k((x))^{m} \backslash\{0\}$.

Problem 2 (Truncation Problem). Given a system $S$ of the form (1),

1. Decide whether or not there exists a non-negative integer sequence $\left(a_{l}\right)_{1 \leq l<\infty}$ such that for any $e \in \mathbb{Z}, l \in \mathbb{Z}^{+}$and column vectors $c_{e}, c_{e+1}, \ldots, c_{e+l-1} \in$ $k^{m}$, the system $S$ possesses a solution $y(x) \in k((x))^{m}$ of the form

$$
y(x)=c_{e} x^{e}+c_{e+1} x^{e+1}+\ldots+c_{e+l-1} x^{e+l-1}+O\left(x^{e+l}\right),
$$

iff the system $S^{\left\langle a_{l}\right\rangle}$ possesses a solution $\tilde{y}(x) \in k((x))^{m}$ such that

$$
\tilde{y}(x)-y(x)=O\left(x^{e+l}\right) .
$$

2. If such sequences $\left(a_{l}\right)$ exist, then find at least one of them.

We suppose that the entries of the matrices (2) are represented algorithmically: for any entry $u(x)$ an algorithm $\Xi_{u}$ (a procedure, terminating in finitely many steps) such that $u(x)=\sum_{i=0}^{\infty} \Xi_{u}(i) x^{i}$ is given. This is factually a model of computation. Our results can be represented without using this model (skipping the undecidability questions then), see Remark 1 
We will show that the existence and truncation problems are algorithmically undecidable in the general case (Section 132). However, both problems can be solved algorithmically for scalar equations (Section 2), and for first order systems (Section 3.2.1). The problems are also solvable for systems of the form (11) with a leading matrix invertible in $\operatorname{Mat}_{m}(k((x)))$ (Section 3.2). Note that we are not able to check algorithmically whether or not a given matrix is invertible. However, if we know in advance that the matrix $A_{r}(x)$ in a given system of the form (11) is invertible and the matrix $A_{0}(x)$ is non-zero then our algorithm completely solves the existence and truncation problems.

The output of our algorithm for the scalar case can be represented as an integer $d \geq-1$ such that

- a solution of $S$ in $k((x)) \backslash\{0\}$ exists iff $d \geq 0$,

- if $d \geq 0$ then a solution of the truncation problem for $S$ is represented by the sequence

$$
a_{l}=\max \{d, l\} \quad(l=1,2, \ldots) .
$$

It follows from (3) that if in the scalar case the truncation problem has a solution then a sequence $\left(a_{l}\right)$ can be taken such that $a_{l}=l$ for all $l$ large enough. In the case of system the situation can be more complicated. However, when systems have invertible leading matrices, we can organise our algorithm in such a way that the output is again an integer $d \geq-1$ such that

- a solution of $S$ in $k((x))^{m} \backslash\{0\}$ exists iff $d \geq 0$,

- if $d \geq 0$ then a solution of the truncation problem for $S$ is represented by the sequence

$$
a_{l}=d+l \quad(l=1,2, \ldots) .
$$

A sequence $\left(a_{l}\right)$ giving a slightly more accurate bound is proposed as well.

At the end of Section 3, we mention another problem that can also be solved using the results of this paper.

To our knowledge, finding sequences $\left(a_{l}\right)$ for the types of systems considered in this paper does not seem to have been done elsewhere in the literature.

\section{The Case of Scalar Equations}

If $m=1$ in the system (11), then this system is a scalar equation. In this particular case, both the existence and truncation problem have an algorithmic solution. The crucial point is that for any such equation we can determine algorithmically the indicial polynomial [12, Ch. IV, § 8] $I_{S}(\lambda) \in k[\lambda] \backslash\{0\}$ such that

(a) if $y(x) \in k((x))^{m}$ is a nonzero solution of $S$ then $I_{S}\left(\operatorname{val}_{x} y(x)\right)=0$, and

(b) if $e^{*}$ is the maximal integer root of $I_{S}(\lambda)$ then $S$ has a solution $y(x) \in k((x))^{m}$ of valuation $e^{*}$. 
The indicial polynomial can be constructed algorithmically, and computer algebra methods for computing its integer roots exist. Supposing in the scalar case that $\min _{i} \operatorname{val}_{x} A_{i}(x)=0$ it is sufficient to know only the coefficients $\left[x^{0}\right] A_{0}(x)$, $\left[x^{0}\right] A_{1}(x), \ldots,\left[x^{0}\right] A_{r}(x)$ for this construction.

Proposition 1. Let $m=1$ in $S$ of the form (1). Let $I_{S}(\lambda)$ be the indicial polynomial of the scalar equation $S$. In this case

(i) If $I_{S}(\lambda)$ has no integer root, then $S$ has no solution in $k((x)) \backslash\{0\}$.

(ii) Otherwise a solution of the truncation problem is given by the sequence

$$
a_{l}=\max \left\{e^{*}-e_{*}+1, l\right\} \quad(l=1,2, \ldots),
$$

where $e_{*}, e^{*}$ are the minimal and maximal integer roots of $I_{S}(\lambda)$, respectively.

Proof. (i) Follows from the property (a) of the indicial equation.

(ii) For an arbitrary solution $y(x) \in k((x)) \backslash\{0\}$ we have $\operatorname{val}_{x} y(x)=e$, where $e$ is an integer root of $I_{S}(x)$. Let a solution have the form $c_{e} x^{e}+c_{e+1} x^{e+1}+\ldots$ Then for any $l>0$ the coefficients $c_{e}, c_{e+1}, \ldots, c_{e+l}$ satisfy a relation $a_{l, 0} c_{e}+a_{l, 1} c_{e+1}+$ $\ldots+a_{l, l} c_{e+l}=0$ where $a_{l, l}=I_{S}(e+l)$ and the constants $a_{l, 0}, a_{l, 1}, \ldots, a_{l, l}$ can be computed from

$$
\left[x^{i}\right] A_{j}(x) \quad(i=0,1, \ldots, l, \quad j=0,1, \ldots, r) .
$$

Thus, if $l>e^{*}-e$ then $c_{e+l}$ is defined uniquely by $c_{e}, c_{e+1}, \ldots, c_{e+l-1}$, and the values (6) define all the values $[e] y(x),[e+1] y(x), \ldots,[e+l] y(x)$ for all belonging to $k((x))$ solutions of $S$ (the Frobenius method [12, Ch. IV, $\S 8]$ ). Observe that the values (6) coincide for $S$ and $S^{\langle l\rangle}$. The claim follows.

The output of the algorithm can be represented in the form of an integer $d \geq-1$ as was explained in the end of Section 1 if $I_{S}(\lambda)$ has no integer root then we set $d=-1$; and $d=e^{*}-e_{*}+1$ otherwise.

Note that this algorithm needs only $\left[x^{0}\right] A_{0}(x),\left[x^{0}\right] A_{1}(x), \ldots,\left[x^{0}\right] A_{r}(x)$ for computing this $d$.

Example 1. Let $k=\mathbb{Q}$, and $S$ be the scalar equation

$$
(1-x) \theta^{2} y+(-2+4 x) \theta y+\left(-x+2 x^{2}+2 x^{3}+2 x^{4}+\ldots\right) y=0,
$$

which has solutions $f(x)=1-x$, and $g(x)=x^{2}-x^{3}$. The indicial polynomial is $\lambda^{2}-2 \lambda$, its roots are 0 and 2 , thus $d=e^{*}-e_{*}+1=3, a_{1}=a_{2}=a_{3}=3$. For finding three terms of each of series solutions we construct $S^{\langle 3\rangle}$

$$
(1-x) \theta^{2} y+(-2+4 x) \theta y+\left(-x+2 x^{2}\right) y=0 .
$$

The latter equation has two independent solutions:

$$
-1+x+0 \cdot x^{2}-x^{3}-\frac{1}{4} x^{4}+O\left(x^{5}\right), \quad-x^{2}+x^{3}+0 \cdot x^{4}+O\left(x^{5}\right) .
$$


We get three wanted terms of solutions of $S$ :

$$
-1+x+0 \cdot x^{2}, \quad-x^{2}+x^{3}+0 \cdot x^{4} .
$$

These polynomials coincide with exact solutions of $S$. Note that we cannot take $S^{\langle 2\rangle}$ for computing two first terms of each of solutions of $S$, since $S^{\langle 2\rangle}$ is

$$
(1-x) \theta^{2} y+(-2+4 x) \theta y-x y=0,
$$

and the space of Laurent solutions of this equation has dimension 1 . We get the solution $-x^{2}+x^{3}+\frac{1}{4} x^{4}+O\left(x^{5}\right)$ which gives us two first terms of $g(x)$, but we do not obtain the corresponding truncation of $f(x)$. This confirms that the sequence (3) is a correct solution of the truncation problem, while the sequence $a_{l}=l$, $l=1,2, \ldots$, is not in general.

Concerning finding integer roots of polynomials over $k$ we can remark the following. If $k=\mathbb{Q}$ then the algorithm is well known. It is also known that if $k_{0}$ is a field of characteristic 0 such that an algorithm for finding integer roots of a polynomials over $k_{0}$ is given then one can find integer roots of polynomials over any simple extension (algebraic or transcendental) of $k_{0}$. This can be used recursively.

\section{The System Case}

As we mentioned in Section 1 we will show that the existence and truncation problems are algorithmically undecidable in the general system case. We will first introduce some auxiliary facts and notions.

\subsection{Undecidability in the General Case}

Many algorithmic problems related to systems are undecidable. To prove this for some of such problems we will use the undecidability of one specific known problem.

We will call a signal any algorithm $\Omega$ computing for each $i=0,1, \ldots$ a value $\Omega(i)$ belonging to $\{0,1\}$ and such that

$-\Omega(0)=1$,

- if $\Omega(i)=0$ for some $i \geq 1$ then $\Omega(i+1)=0$.

A signal $\Omega$ is infinite if $\Omega(i)=1$ for all $i \geq 0$ and finite otherwise.

We will use the following fact:

The problem of recognizing whether or not a given signal is finite is undecidable.

This is a consequence of classical Turing's result on undecidability of the problem of terminating of an algorithm [16]. 
Using this fact we can prove that the problem of recognizing whether or not a given non-zero square matrix with entries in $k((x))$ is invertible over the field $k((x))$. Indeed, let $k=\mathbb{Q}$, and $\Omega$ be an arbitrary signal. The matrix

$$
\left(\begin{array}{cc}
1-x & 1 \\
1 & \sum_{i=0}^{\infty} \Omega(i) x^{i}
\end{array}\right)
$$

is invertible iff the signal $\Omega$ is finite.

If $\Omega$ is a signal and $s(x)=s_{0}+s_{1} x+s_{2} x^{2}+\ldots \in k[[x]]$ then we set $\Omega * s(x)=$ $\Omega(0) s_{0}+\Omega(1) s_{1} x+\Omega(2) s_{2} x^{2}+\ldots$; given a system $S$ of the form (11), we can replace any entry $s(x)$ of the matrix coefficients of $S$ by $\Omega * s(x)$, the obtained new system will be denoted $\Omega * S$.

Proposition 2. (i) The algorithmic problem of recognizing whether or not a given system of the form (1) has a solution in $k((x))^{m} \backslash\{0\}$ is undecidable.

(ii) There exist systems of the form (1) for which the truncation problem has no solution (no sequence $\left(a_{i}\right)$ exists); the algorithmic problem of recognizing whether or not the truncation problem for a given system of the form (1) has a solution (a sequence $\left(a_{i}\right)$ ) is undecidable.

Proof. (i) Let $S$ be the system

$$
A(x) x \theta y+A(x) y=0
$$

where $A(x)$ is the matrix (7). Then the system $\Omega * S$ has a solution in $k((x))^{2} \backslash\{0\}$ iff the signal $\Omega$ is infinite. Indeed, if the matrix (7) is invertible then the system is equivalent to $\left(\begin{array}{ll}x & 0 \\ 0 & x\end{array}\right) \theta y+\left(\begin{array}{ll}1 & 0 \\ 0 & 1\end{array}\right) y=0$ which has no non-zero solution in $k((x))^{2}$. But if (7) is not invertible then the system has solutions in $k((x))^{m} \backslash\{0\}$ since the system $\left(\begin{array}{ll}x & 0 \\ 0 & x\end{array}\right) \theta y+\left(\begin{array}{ll}1 & 0 \\ 0 & 1\end{array}\right) y=\left(\begin{array}{l}s_{1} \\ s_{2}\end{array}\right)$ with $s_{1}(x), s_{2}(x) \in k((x))$ has a solution in $k((x))^{2} \backslash\{0\}$ if at least one of the series $s_{1}(x), s_{2}(x)$ is non-zero.

(ii) It follows from the proof of (i) that no sequence $\left(a_{i}\right)$ exists for the system $S$ of the form (8). However, if $\Omega$ is a finite signal then any sequence $\left(a_{l}\right)$ can be used for the system $\Omega * S$ because neither $\Omega * S$ nor its truncations have solutions in $k((x)) \backslash\{0\}$. So for the system $\Omega * S$ a sequence $\left(a_{l}\right)$ exists iff $\Omega$ is finite.

Remark 1. As it was mentioned in the Introduction, our results can be formulated without a special supposition on the form of series representation. It follows from the proof of Proposition 圆 that for some systems of the form (11) no sequence $\left(a_{l}\right)$ exists. In Section 3.2 we will show that if the leading matrix is invertible in $\operatorname{Mat}_{m}(k((x)))$, then after a finite number of steps we can recognize whether or not this system has a solution in $k((x))^{m} \backslash\{0\}$. If the answer is affirmative then there is a guarantee that a sequence $\left(a_{l}\right)$ exists and can be presented.

\subsection{Some Particular Decidable Cases}

We will now show that in some particular cases the existence and truncation problems are decidable. 


\section{2,1 First Order Systems}

In this section, we consider first order systems of the form

$$
\theta y=A(x) y,
$$

where $A(x) \in \operatorname{Mat}_{m}(k((x)))$. We will show that the existence and truncations problems are decidable for systems of this form. Our approach is based on the concept of simple systems [3] (which is related to the notion of super-irreducible forms of linear differential systems [13]).

A system $\theta y=A(x) y$ can always be rewritten as a system of the form

$$
D(x) \theta y=N(x) y
$$

where $D(x), N(x) \in \operatorname{Mat}_{m}(k[[x]])$ with $\min \left\{\operatorname{val}_{x} D(x), \operatorname{val}_{x} N(x)\right\}=0$.

For this take $N(x)=D(x) A(x)$ and $D(x)=\operatorname{diag}\left(x^{\alpha_{1}}, x^{\alpha_{2}}, \ldots, x^{\alpha_{m}}\right)$ where $\alpha_{i}=\max \left\{0,-\operatorname{val}_{x} A_{i .}(x)\right\}$, here $A_{i .}(x)$ denotes the $i$ th row of the matrix $A(x)$.

Now consider a general system $S$ of the form (10) with coefficients $D(x)=$ $\sum_{\lambda}^{\infty} D_{i=0} D_{i}, N(x)=\sum_{0}^{\infty} N_{i} x^{i}$. With $S$ we associate the following polynomial in

$$
I_{S}(\lambda)=\operatorname{det}\left(D_{0} \lambda-N_{0}\right) .
$$

If this polynomial $I_{S}(\lambda)$ is non-zero then we shall say that the system $S$ is simple and refer to the polynomial $I_{S}(\lambda)$ as the indicial polynomial of $S$ as in 7 , Definition 2.1]. By extension, a system of the form (9) will be called simple if the corresponding system (10) is simple.

The following result shows that for simple systems, the existence and truncation problems can be solved very similarly as in the scalar case.

Proposition 3. Given a simple system of the form (10) with indicial polynomial $I_{S}(\lambda)$ given by (11), we have

(i) If $I_{S}(\lambda)$ has no integer root, then $S$ has no solution in $k((x)) \backslash\{0\}$.

(ii) Otherwise a solution of the truncation problem is given by the sequence

$$
a_{l}=\max \left\{e^{*}-e_{*}+1, l\right\} \quad(l=1,2, \ldots),
$$

where $e_{*}$ and $e^{*}$ are the minimal and maximal integer roots of $I_{S}(\lambda)$, respectively.

Proof. (i) The statements of this proposition follow from the results in [7. The algorithm presented therein computes regular formal solutions (i.e. in particular, Laurent series solutions) by finding successive terms. The equation that determines a coefficient of the monomial $x^{j}(j \geq 0)$, part of a solution $y=x^{e} \sum y_{j} x^{j}$ $\left(y_{0} \neq 0\right)$, is

$$
\left((e+j) D_{0}-N_{0}\right) y_{j}=-b_{j}
$$

where $b_{0}=0$ and

$$
b_{j}=\left((e+j-1) D_{1}-N_{1}\right) y_{j-1}+\cdots+\left(e D_{j}-N_{j}\right) y_{0} . \quad(j \geq 1)
$$


Putting $j=0$, we see why the nonzero polynomial $I_{S}(\lambda)=\operatorname{det}\left(\lambda D_{0}-N_{0}\right)$ plays a similar role as the indicial polynomial in the scalar case (see Section 2): We have $I_{S}\left(\operatorname{val}_{x} y\right)=0$ so the existence of integer roots of $I_{S}$ is a necessary condition for the existence of solutions in $k((x))^{m} \backslash\{0\}$.

(ii) Let $x^{e} \sum y_{j} x^{j}$ be a solution of $D^{\left\langle a_{l}\right\rangle}(x) \theta y=N^{\left\langle a_{l}\right\rangle}(x)$ where $a_{l}=\max \left\{e^{*}-\right.$ $\left.e_{*}+1, l\right\}$. This means that equations (13) are satisfied for $j=0,1, \ldots, l$. In order to extend this to a solution of the untruncated system, we need to compute the coefficient $y_{j}$ for $j>l$, we note that the matrix $(e+j) D_{0}-N_{0}$ is invertible since $e+j>e+l \geq e^{*}$. This means that we can determine $y_{j}$ uniquely.

Example 2. Let $k=\mathbb{Q}$ and $S$ be the first-order system

$$
\theta y=\left(\begin{array}{cc}
0 & x^{3} \\
0 & -3
\end{array}\right) y
$$

which has as a basis of solutions

$$
y_{1}(x)=\left(\begin{array}{l}
1 \\
0
\end{array}\right) \quad y_{2}(x)=\left(\begin{array}{c}
\ln (x) \\
x^{-3}
\end{array}\right) .
$$

Hence the space of solution of $S$ in $k((x))^{2}$ has dimension 1. This system is simple: it is already in the form (10) with $D(x)=I_{2}$ and $N(x)=\left(\begin{array}{cc}0 & x^{3} \\ 0 & -3\end{array}\right)$. Its indicial polynomial is

$$
I_{S}(\lambda)=\left|\begin{array}{cc}
\lambda & 0 \\
0 & \lambda+3
\end{array}\right|=\lambda(\lambda+3)
$$

The roots of $I_{S}(\lambda)$ are -3 and 0 , thus $d=e^{*}-e_{*}+1=4, a_{1}=a_{2}=a_{3}=4$. For $l=1,2,3$ the l-truncation of $S$ is

$$
\theta y=\left(\begin{array}{cc}
0 & 0 \\
0 & -3
\end{array}\right) y .
$$

The latter system has two independent solutions in $k((x))^{2}$ :

$$
\tilde{y}_{1}(x)=\left(\begin{array}{l}
1 \\
0
\end{array}\right) \quad \tilde{y}_{2}(x)=\left(\begin{array}{c}
0 \\
x^{-3}
\end{array}\right) .
$$

This confirms that the sequence (12) is a correct solution of the truncation problem, while the sequence $a_{l}=l, l=1,2, \ldots$, is not in general.

Remark 2. The results in Proposition 3 are valid for more general class of systems, namely simple systems of higher order [4], [5]. Recall that a system $S$ of the form (1) is simple if the matrix polynomial defined by

$$
L_{S}(\lambda)=A_{r}(0) \lambda^{r}+A_{r-1}(0) \lambda^{r-1}+\cdots+A_{0}(0),
$$

is regular, i.e., $\operatorname{det}\left(L_{S}(\lambda)\right) \not \equiv 0$. For a simple system $S$ we define its indicial polynomial as $I_{S}(\lambda)=\operatorname{det}\left(L_{S}(\lambda)\right)$. 
Having clarified the situation if a system is simple, we now solve Problem 1 and Problem 2 for the case of a general first order system of the form (9). Define the span of an invertible matrix $T(x) \in \operatorname{Mat}_{m}(k((x)))$ by

$$
\sigma(T(x))=-\operatorname{val}_{x} T(x)-\operatorname{val}_{x} T^{-1}(x) .
$$

We need the following well-known technical lemma, which has been stated in [14, see also its use in [15].

Lemma 1. ([14]) Let $A(x), T(x) \in$ Mat $_{m}(k((x)))$ with $T(x)$ invertible and let $\tilde{A}(x)=T^{-1}(x)(A(x) T(x)-\theta(T(x)))$. Then the coefficient $\tilde{A}_{j}$ depends only on the $A_{i}$ with $i \leq j+\sigma(T(x))$.

Proposition 4. Consider a system $\theta y=A(x) y$ of the form (9), and let $q=$ $\max \left\{0,-\operatorname{val}_{x} A(x)\right\}$.

(i) There exists an algorithm, using only the first $m q$ terms of the entries of $x^{q} A(x)$, that computes an invertible matrix $T(x) \in \operatorname{Mat}_{m}(k[x])$ with $\operatorname{det} T(x)=$ $c x^{\nu}$ for some nonzero constant $c \in k$ and some nonnegative integer $\nu$, with span $\sigma(T(x)) \leq(m-1) q$ such that the substitution $y=T(x) z$ yields a system

$$
\theta z=B(x) z
$$

which is simple. Let $\tilde{I}(\lambda)$ denote the indicial polynomial of the corresponding simple system.

(ii) If $\tilde{I}(\lambda)$ has no integer root then (9) has no solution in $k((x))^{m} \backslash\{0\}$.

(iii) Otherwise a solution of the truncation problem (for the input system (9)), is given by the sequence

$$
a_{l}=m q+\max \left\{e^{*}-e_{*}+1, l+(m-1) q\right\}, \quad(l=1,2, \ldots)
$$

where $e_{*}, e^{*}$ are the minimal and maximal integer roots of $\tilde{I}(\lambda)$, respectively.

Proof. (i) The algorithm from [13] computes the so-called super-irreducible form of a given system (9). It was shown in 3 , that if a system has the super-irreducible form then it can be written as a simple system. The algorithm from [13] needs at the most $(m-1) q$ reduction steps (see, for example, the proof of Proposition 2.2 in [10]). At each step, a transformation matrix with span 1 is computed. Overall, this shows the estimate on the span of $T(x)$.

(ii) Compute an invertible matrix $T(x) \in \operatorname{Mat}_{m}(k[x])$ such that the matrix

$$
B(x)=T^{-1}(x) A(x) T(x)-T^{-1}(x) \theta T(x)
$$

defines the system (14). Write (14) as a simple system $D(x) \theta z=N(x) z$, and let $\tilde{I}(\lambda)=\operatorname{det}\left(N_{0}-\lambda D_{0}\right)$ be its indicial polynomial. If $\tilde{I}(\lambda)$ does not have integer roots, the simple system does not have any solutions in $k((x))^{m} \backslash\{0\}$. Hence the original system (9) cannot have solutions of this form either.

(iii) In order to solve the truncation problem for the input system, note that due to the relationship $y=T(x) z$ between solutions $y$ of the input system and 
$z$ of the simple system, we have to compute at the most $l+\sigma(T(x))$ terms of $z$, if we need $l$ terms of $y$. Using Proposition 3 to first solve the truncation problem for the simple system $D(x) \theta z=N(x) z$, we obtain $\tilde{a}_{l}=\max \left\{e^{*}-\right.$ $\left.e_{*}+1, l+\sigma(T(x))\right\}$. It then remains to show how many terms of the input system are required in order to ensure that we have $\tilde{a}_{l}$ terms of the simple system. This can be seen as follows: for any $j \geq 0$, the coefficients $D_{j}$ and $N_{j}$ of the simple system depend on the coefficients $B_{0}, \ldots, B_{q(B)-1+j}$ of the matrix $B(x)$, due to the construction of $D(x)$. Here, $q(B)=\max \left(0,-\operatorname{val}_{x}(B(x))\right.$. Using Lemma 1, the coefficient $B_{q(B)-1+j}$ depends only on the coefficients $A_{i}$ with $i \leq j+q(B)-1+\sigma(T(x))$ of $A(x)$. The proof is completed by the fact that $\sigma(T(x)) \leq(m-1) q$ for the transformation matrix $T(x)$ computed by the superreduction algorithm as shown in (i), and that $q(B) \leq q$.

Example 3. Let $k=\mathbb{Q}, q$ be a positive integer and $S$ be the first-order system

$$
\theta y=A(x) y \text { where } A(x)=\left(\begin{array}{cc}
x^{4} & x^{3-q} \\
-x^{q+5}-4 x^{q+1} & -x^{4}+(q-3)
\end{array}\right),
$$

which has as a basis of solutions

$$
y_{1}(x)=\left(\begin{array}{c}
1 \\
-x^{q+1}
\end{array}\right) \quad y_{2}(x)=\left(\begin{array}{c}
\ln (x) \\
-x^{q+1} \ln (x)-x^{q-3}
\end{array}\right) .
$$

Hence the space of solution of $S$ in $k((x))^{2}$ has dimension 1 . This system $S$ is not simple. Let $T(x)=\left(\begin{array}{ll}1 & 0 \\ 0 & x^{q}\end{array}\right)$ of span $q$. Then the substitution $y=T(x) z$ yields the equivalent system

$$
\theta z=B(x) z \text { where } \quad B(x)=\left(\begin{array}{cc}
x^{4} & x^{3} \\
-x^{5}-4 x & -x^{4}-3
\end{array}\right) .
$$

The latter system is simple, and its indicial polynomial is

$$
\tilde{I}(\lambda)=\left|\begin{array}{ll}
\lambda & 0 \\
0 & \lambda+3
\end{array}\right|=\lambda(\lambda+3) .
$$

The roots of $\tilde{I}(\lambda)$ are -3 and 0 , thus $e^{*}-e_{*}+1=4, a_{1}=a_{2}=a_{3}=2 q+4$ and $a_{l}=2 q+l$ for $l \geq 4$. Take, for example, $q=3$. Then for $l=4,5,6,7$ the l-truncation of $S$ is

$$
\theta y=\left(\begin{array}{ll}
0 & 1 \\
0 & 0
\end{array}\right) y \text {. }
$$

The latter system has one independent solution in $k((x))^{2}$, namely $\left(\begin{array}{l}1 \\ 0\end{array}\right)$.

\section{2,2 Extension to Higher Order Systems}

The results from the previous section can be easily extended to a system of the form

$$
\theta^{r} y=-U_{r-1}(x) \theta^{r-1} y-\cdots-U_{0}(x) y
$$


where $U_{0}(x), \ldots, U_{r-1}(x) \in \operatorname{Mat}_{m}(k((x)))$. The idea is that there exists a linear first order system $\theta Y(x)=U(x) Y(x)$ with companion block matrix $U(x)$ that corresponds to (16). This matrix belongs to $\operatorname{Mat}_{n}(k((x))), n=r m$ :

$$
U(x)=\left(\begin{array}{cccc}
0 & I_{m} & \ldots & 0 \\
\ldots & \ldots & \ldots & \ldots \\
0 & 0 & \ldots & I_{m} \\
-U_{0}(x) & -U_{1}(x) & \ldots & -U_{r-1}(x)
\end{array}\right) .
$$

Applying Proposition 4 to system $\theta Y(x)=U(x) Y(x)$ we obtain the following proposition:

Proposition 5. Let $q=\max \left\{0,-\operatorname{val}_{x} U_{r-1}(x), \ldots,-\operatorname{val}_{x} U_{0}(x)\right\}$. There exists an algorithm, that uses only the first rmq terms (i.e., terms of degree less than rmq) of the matrices $x^{q} U_{0}(x), \ldots, x^{q} U_{r-1}(x)$, and computes a nonzero polynomial $\tilde{I}(\lambda)$ such that:

- if $\tilde{I}(\lambda)$ has no integer root then (16) has no solution in $k((x))^{m} \backslash\{0\}$,

- otherwise a solution of the truncation problem is given by the sequence

$$
a_{l}=r m q+\max \left\{e^{*}-e_{*}+1, l+(r m-1) q\right\} \quad(l=1,2, \ldots),
$$

where $e_{*}, e^{*}$ are the minimal and maximal integer roots of $\tilde{I}(\lambda)$, respectively.

\section{2,3 Systems with Invertible Leading Matrices}

Let the leading matrix of a system $S$ of the form (1) be invertible. In this case $S$ can be rewritten as the system $\bar{S}$ of the form

$$
\theta^{r} y=-A_{r}^{-1}(x) A_{r-1}(x) \theta^{r-1} y-\cdots-A_{r}^{-1}(x) A_{0}(x) y .
$$

Let

$$
\gamma=\min _{i} \operatorname{val}_{x}\left(A_{r}^{-1}(x) A_{i}(x)\right),
$$

and $q=\max \{-\gamma, 0\}$. The question to be answered is: given a non-negative integer $v$, how many first terms of the entries of $S$ do we need to compute $v$ first terms of

$$
x^{q} A_{r}^{-1}(x) A_{r-1}(x), \quad x^{q} A_{r}^{-1}(x) A_{r-2}(x), \quad \ldots, \quad x^{q} A_{r}^{-1}(x) A_{0}(x) ?
$$

Before answering this question we formulate a few facts related to the operations which we use to transform $S$ to $\bar{S}$. As before, we suppose that all power series are represented algorithmically.

(A) Let it be known in advance that amongst the given series

$$
s_{1}(x), s_{2}(x), \ldots, s_{p}(x) \in k[[x]], \quad p \geq 1,
$$

there is at least one non-zero. Then we can compute

$$
\nu=\min _{i} \operatorname{val}_{x} s_{i}(x) .
$$

To do this we consider the series $s_{1}(x), s_{2}(x), \ldots, s_{p}(x)$ "in parallel": we generate algorithmically the sequence 


$$
\left[x^{0}\right] s_{1}(x), \ldots,\left[x^{0}\right] s_{p}(x),\left[x^{1}\right] s_{1}(x), \ldots,\left[x^{1}\right] s_{p}(x), \ldots
$$

until we find $i$ such that $\left[x^{i}\right] s_{j}(x) \neq 0$ for some $1 \leq j \leq p$. Then $\nu=i$.

(B) Let it be known in advance that among given matrices

$$
M_{1}(x), M_{2}(x), \ldots, M_{p}(x) \in \operatorname{Mat}(k[[x]]), \quad p \geq 1,
$$

there is at least one non-zero. Then we can compute $\min _{i} \operatorname{val}_{x} M_{i}(x)$. To do this we consider the entries of all the matrices "in parallel" (as in (A)).

(C) Let it be known in advance that a matrix $M(x) \in \operatorname{Mat}(k[[x]])$ is invertible. We can compute $\operatorname{val}_{x} \operatorname{det} M(x)$, using $\operatorname{val}_{x} \operatorname{det} M(x)+1$ initial entries of the matrix $M$. We can also compute $\operatorname{val}_{x} M^{-1}(x)$ which is equal to the difference of the minimum of the valuation of all co-factors of $M(x)$ and $\operatorname{val}_{x} \operatorname{det} M(x)$. This difference is non-positive, thus, we use $\operatorname{val}_{x} \operatorname{det} M(x)+1$ initial terms of the entries of the matrix $M(x)$.

Every time when below in $\left(\mathrm{A}^{\prime}\right),\left(\mathrm{B}^{\prime}\right),\left(\mathrm{C}^{\prime}\right)$ and in Proposition 6 we tell about the first $w$ terms (where $w$ is a positive integer) of entries of some matrices belonging to $k[[x]]$, we have in mind the terms of degree less than $w$.

We get from $(\mathrm{A}),(\mathrm{B}),(\mathrm{C})$ the following.

$\left(\mathrm{A}^{\prime}\right)$ We use $\operatorname{val}_{x} \operatorname{det} A_{r}(x)+1$ first terms of the entries of the matrix $A_{r}(x)$ to compute val $\operatorname{det} A_{r}(x)$ and $\operatorname{val}_{x} A_{r}^{-1}(x)$.

$\left(\mathrm{B}^{\prime}\right)$ We use no more than $\operatorname{val}_{x} \operatorname{det} A_{r}(x)+\gamma+1$ first terms of the entries of the matrices $A_{0}(x), A_{1}(x), \ldots, A_{r}(x)$ to compute $\gamma$ (see (20)).

$\left(\mathrm{C}^{\prime}\right)$ We use no more than $\operatorname{val}_{x} \operatorname{det} A_{r}(x)+\gamma+v$ first terms of the entries of the matrices $A_{0}(x), A_{1}(x), \ldots, A_{r}(x)$ to compute the first $v$ terms of (21).

This and Proposition 5 imply the following statement related to systems of the form (11) with invertible $A_{r}(x)$.

Proposition 6. Let $\gamma$ be as in (20) and $q=\max \{-\gamma, 0\}$. There exists an algorithm, that uses only the first

$$
r m q+\gamma+\operatorname{val}_{x} \operatorname{det} A_{r}(x)+1
$$

terms of the entries of the matrices $A_{0}(x), A_{1}(x), \ldots, A_{r}(x)$, and computes a nonzero polynomial $\tilde{I}(\lambda)$ such that:

- if $\tilde{I}(\lambda)$ has no integer root then (1) has no solution in $k((x))^{m} \backslash\{0\}$,

- otherwise a solution of the truncation problem is given by the sequence

$$
a_{l}=r m q+\gamma+\operatorname{val}_{x} \operatorname{det} A_{r}(x)+\max \left\{e^{*}-e_{*}+1, l+(r m-1) q\right\} \quad(l=1,2, \ldots),
$$

where $e_{*}, e^{*}$ are the minimal and maximal integer roots of $\tilde{I}(\lambda)$, respectively.

Finally we can formulate a consequence of the latter proposition:

Proposition 7. For a given system $S$ of the form (1) with invertible $A_{r}(x)$ we can compute algorithmically an integer $d \geq-1$ such that 
- a solution of $S$ in $k((x))^{m} \backslash\{0\}$ exists iff $d \geq 0$,

- if $d \geq 0$ then a solution of the truncation problem for $S$ is represented by the sequence $a_{l}=d+l, l=1,2, \ldots$

Proof. Indeed, we set $d=-1$ if the polynomial $\tilde{I}(\lambda)$ has no integer root and $d=2 r m q-q+\gamma+\operatorname{val}_{x} \operatorname{det} A_{r}(x)+e^{*}-e_{*}+1$ otherwise, where $q, \gamma, \tilde{I}_{S}(\lambda)$, $e^{*}, e_{*}$ are as in Proposition 6 .

The following example shows that unlike the scalar case in the case of system we cannot in general take a sequence $\left(a_{l}\right)$ such that $a_{l}=l$ at least for all $l$ large enough.

Example 4. Consider the system $x \theta y=A(x) y$ where

$$
A(x)=\left(\begin{array}{cc}
0 & 1 \\
x^{2} u(x) & 0
\end{array}\right)
$$

$u(x)=x+x^{2}+x^{3}+\ldots, y=\left(\begin{array}{l}y_{1} \\ y_{2}\end{array}\right)$. It is easy to show that $y_{1}$ satisfies the equation

$$
\theta^{2} y_{1}+\theta y_{1}-u(x) y_{1}=0 .
$$

For the latter equation the sequence $b_{l}=l, l=1,2, \ldots$, is a solution of the truncation problem. For any other solution $\left(b_{l}^{\prime}\right)$ of this problem we will have $b_{l}^{\prime} \geq b_{l}, l=1,2, \ldots$ Note that $l$-truncation of the original system $x \theta y=A(x) y$ induces $(l-2)$-truncation of (23). However, the sequence $c_{l}=l-2, l=1,2, \ldots$, is not a solution of the truncation problem for (23). Thus, a sequence $\left(a_{l}\right)$ which is a solution of the truncated problem for the original system must be such that $a_{l} \geq l+2, l=1,2, \ldots$ If we replace in the original system $x^{2}$ by $x^{2 q}$ with integer $q>1$, then we will obtain $a_{l} \geq l+2 q, l=1,2, \ldots$

Our results can be also used for solving the following problem. Suppose that for a system $S$ of the form (11) only a finite number of terms of the entries of $A_{0}(x), A_{1}(x), \ldots, A_{r}(x)$ is known. So we know not the system $S$ itself but the system $S^{\langle v\rangle}$ for some non-negative integer $v$. Suppose that we also know that $A_{r}(x)$ is invertible and that $S$ has solutions in $k((x))^{m} \backslash\{0\}$. How many terms of these solutions can be determined from the given "approximate" system $S^{\langle v\rangle}$ ? Some non-trivial lower bound can be obtained from Propositions 1, 5, and 6.

\section{Implementation}

We have used the results obtained in this paper to improve some functionality contained in the Maple package ISOLDE [11. The RegularSolutions function computes formal regular solutions of first order linear functional systems such as systems of linear differential, difference, and $q$-difference equations. In particular, it can be used for computing truncated Laurent series solutions of first order linear differential systems. 
The old implementation used the sequence $a_{l}=l$ and did hence not always compute the accurate space of truncated Laurent series solutions, as the following example shows:

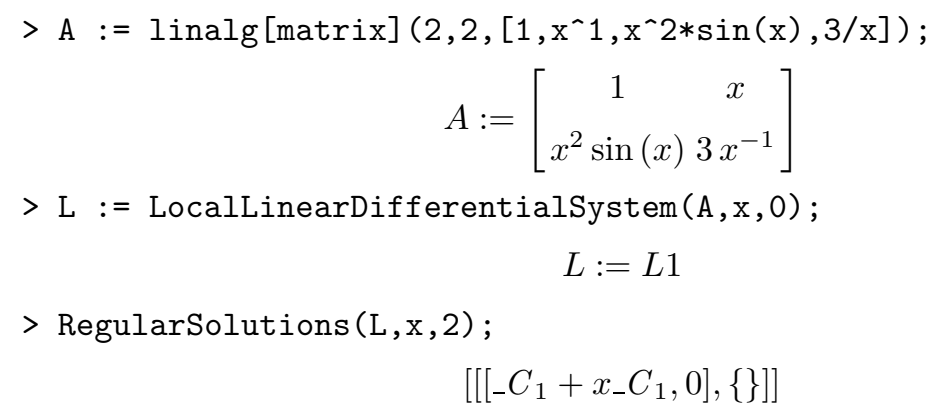

Here, for $l=2$, the function returns only one truncated Laurent series. We have added the new option 'allSolutions' which ensures that a complete basis of the regular solutions space is computed, by taking into account formula (15). The sequence is then $a_{1}=a_{2}=3$ and $a_{l}=l$ for $l \geq 3$, since the indicial polynomial of the system has roots 0 and 3 . The output is then

> RegularSolutions (L, x,2, 'allSolutions');

$$
\text { [[[_ } \left.\left.\left.C_{1}+x_{-} C_{1}, x^{3}{ }_{-} C_{2}\right],\{\}\right]\right]
$$

This new feature will be available in the upcoming new release of ISOLDE.

\section{Conclusion}

In this paper, we have investigated the existence and truncation problem for higher-order linear differential systems. We have shown that they are undecidable in the general case but they can be solved in the case of the system's leading matrix being invertible. In the decidable cases, this means that we can reduce the problem of finding Laurent series solutions of systems with power series coefficients to that of finding the same type of solutions for systems with polynomial coefficients. A number of methods exist to do this task efficiently (e.g., 16]).

The mathematical techniques we employ in this paper use the algebra of polynomials and matrices, and we give explicit formulae for finding $a_{l}$ for a given $l$. An implementation of our results can be done easily in any computer algebra system, as demonstrated in the previous section for the Maple package ISOLDE, and this equally applies to the implementations of the algorithms from [16]. We hope that this paper hence also makes a practical contribution to the scientific computing community, wishing to use computer algebra for handling systems of linear differential equations.

From our work, new questions arise. For example, can we solve the existence and truncation problem when we know in advance that the equations of a given system are independent over $k((x))[\theta]$ while the leading matrix is not invertible? Can our results be extended to more general classes of equations, such as difference and $q$-difference systems? We will continue to investigate this line of enquiry. 


\section{References}

1. Abramov, S., Bronstein, M., Khmelnov, D.: On regular and logarithmic solutions of ordinary linear differential systems. In: Ganzha, V.G., Mayr, E.W., Vorozhtsov, E.V. (eds.) CASC 2005. LNCS, vol. 3718, pp. 1-12. Springer, Heidelberg (2005)

2. Barkatou, M.A.: A rational version of Moser's Algorithm. In: ISSAC 1995 Proceedings, pp. 297-302. ACM Press, New York (1995)

3. Barkatou, M.A.: On rational solutions of systems of linear differential equations. J. Symbolic Computation 28, 547-567 (1999)

4. Barkatou, M.A., Cluzeau, T., El Bacha, C.: Algorithms for regular solutions of higher-order linear differential systems. In: Johnson, J.R., Park, H., Kaltofen, E. (eds.) ISSAC 2009 Proceedings, pp. 7-14. ACM Press, New York (2009)

5. Barkatou, M.A., Cluzeau, T., El Bacha, C.: Simple forms of higher-order linear differential systems and their applications to computing regular solutions. J. Symbolic Computation 46, 633-658 (2011)

6. Barkatou, M.A., El Bacha, C., Pflügel, E.: Simultaneously row- and columnreduced higher-order linear differential systems. In: Koepf, W. (ed.) ISSAC 2010 Proceedings, pp. 45-52. ACM Press, New York (2010)

7. Barkatou, M.A., Pflügel, E.: An algorithm computing the regular formal solutions of a system of linear differential equations. J. Symbolic Computation 28, 569-588 (1999)

8. Barkatou, M.A., Pflügel, E.: On the equivalence problem of linear differential systems and its application for factoring completely reducible systems. In: Gloor, O. (ed.) ISSAC 1998 Proceedings, pp. 268-275. ACM Press, New York (1998)

9. Barkatou, M.A., Pflügel, E.: Computing super-irreducible forms of systems of linear differential equations via Moser-reduction: A new approach. In: Dongming, W. (ed.) ISSAC 2007 Proceedings, pp. 1-8. ACM Press, New York (2007)

10. Barkatou, M.A., Pflügel, E.: On the Moser- and super-reduction algorithms of systems of linear differential equations and their complexity. J. Symbolic Computation 44, 1017-1036 (2009)

11. Barkatou, M.A., Pflügel, E.: The ISOLDE package. A SourceForge Open Source project (2006), http://isolde.sourceforge.net

12. Coddington, E., Levinson, N.: Theory of Ordinary Differential Equations. McGrawHill, New York (1955)

13. Hilali, A., Wazner, A.: Formes super-irréductibles des systèmes différentiels linéaires. Numer. Math. 50, 429-449 (1987)

14. Lutz, D.A., Schäfke, R.: On the identification and stability of formal invariants for singular differential equations. Linear Algebra and Its Applications 72, 1-46 (1985)

15. Pflügel, E.: Effective formal reduction of linear differential systems. Applicable Algebra in Engineering, Communication and Computation 10, 153-187 (2000)

16. Turing, A.: On computable numbers, with an application to the Entscheidungsproblem. Proceedings of the London Mathematical Society, Series 2 42, 230-265 (1936)

17. Maple online help: http://www .maplesoft.com/support/help/ 